\title{
Einmalige, hochdosierte Vitamin-D-Injektion lindert Beschwerden!
}

Fragestellung: Führt eine einzelne hochdosierte intramuskuläre Injektion von 600000 IE Vitamin D zu einer Besserung der diabetischen Neuropathie?

Hintergrund: Etwa jeder fünfte Mensch mit Diabetes leidet an einer Neuropathie mit symmetrischen Parästhesien, Dysästhesien, lanzinierenden Schmerzen und Berührungsempfindlichkeit, die nachts zunehmen und Schlafstörungen verursachen. Schon aus der Vielzahl der propagierten Empfehlungen kann man ableiten, dass es keine zuverlässige Therapie gibt. Beobachtungsstudien und Metaanalysen haben eine Korrelation zwischen Vitamin-D-Spiegeln und klinischen wie elektrophysiologischen neurologischen Defiziten gezeigt. Trotz einer Vielzahl von experimentellen Daten fehlen dazu aber klinische Studien.

Patienten und Methoden: In die Studie wurden 143 Diabetespatienten - vorwiegend Typ 2 - mit schwerer schmerzhafter Neuropathie aufgenommen. Stärke und Qualität der Schmerzen wurden mit drei verschiedenen Scores vor Vitamin-D-Gabe und dann in Abständen von etwa fünf Wochen viermal bestimmt, ferner diverse Laborparameter einschließlich der Vitamin-D-Spiegel vor und

\section{Originalie}

Basit A, Basit K A, Fawwad A. et al. Vitamin $D$ for the treatment of painful diabetic neuropathy. BMJ Open Diab. Res. Care 2016:4:e0001 48. nach der Vitamin-D-Gabe.

Ergebnisse: Art, Stärke und Qualität der Schmerzen nahmen nach 20 Wochen bei allen drei Scores erheblich und jeweils signifikant $a b(\mathrm{p}<$ 0,0001). Die Besserung war bereits nach 8 Wochen deutlich erkennbar und verstärkte sichbis zum Ende der 20. Woche. Die Vitamin-D-Spiegel stiegen in diesem Zeitraum von 31,7 auf 46,2 ng/ml an. Allerdings war die Besserung der Symptome bei Patienten mit niedrigen, grenzwertigen und normalen Vitamin-D-Ausgangswerten gleich.

Schlussfolgerung: Die hochdosierte, einmalige, intramuskuläre Gabe von Vitamin D führt zu einer deutlichen Besserung der schmerzhaften diabetischen Neuropathie.

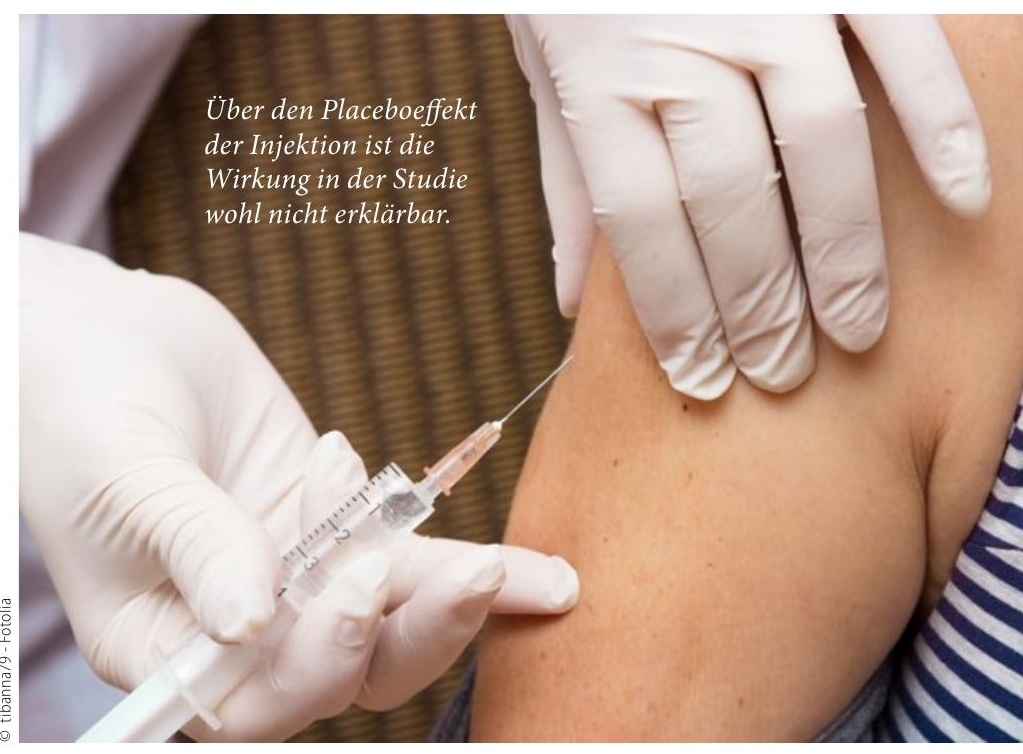

\section{- Kommentar von Prof. Dr. med. Heinrich Holzgreve}

\section{Schwer erklärbarer Therapieerfolg}

Die Besserung der Beschwerden ist bemerkenswert, zumal sie mit den Vitamin-D-Ausgangswerten der Patienten nicht korreliert, also die Studienhypothese nicht bestätigt. Die Autoren weisen selbst darauf hin, dass es sich um eine Beobachtungstudie ohne Kontrollgruppe und ohne objektiv messbare Erfolgskriterien handelt. Allerdings erwartet man von einem Placeboeffekt eine schnelle und kurzlebige Wirkung, nicht aber eine derartige allmähliche, konstante Verstärkung bis zu einer Dauer von 20 Wochen.

Die diabetische Neuropathie gilt als schwer behandelbar bis therapieresistent. Die in den Leitlinien empfohlenen Maßnahmen sind nur begrenzt wirksam und teils nebenwirkungsträchtig. Die Vitamin-D-Gabe ist dagegen attraktiv: einmalig, sicher und möglicherweise mindestens 20 Wochen wirksam. Eine kontrollierte Studie über längere Dauer ist wünschenswert.

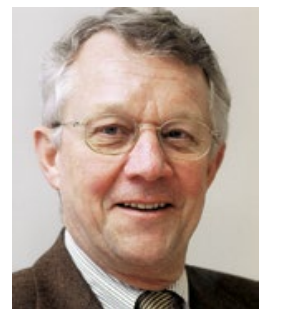

Prof. Dr. med. Heinrich Holzgreve

Internist, Kardiologische Praxis

Burgstr. 7, 80331 München

h.holzgreve@t-online.de 